Ann. Sci. forest., 1972, 29 (1), 35-48.

\title{
MÉTHODE UTILISÉE POUR LA CONSTRUCTION RAPIDE DE TABLES DE PRODUCTION PROVISOIRES EN FRANCE
}

\author{
N. DECOURT \\ Station de Sylviculture et de Production, \\ Centre national de Recherches forestières, I.N.R.A., \\ 54 - Champenoux, par Einville
}

\begin{abstract}
RÉSUMÉ
La méthode utilise les données récoltées dans des placettes temporaires sans mesure directe d'accroissement en volume. Les peuplements sont décrits par des relations mathématiques, comme également la croissance en hauteur en fonction de la station. La sylviculture est définie de la même façon. Les relations sont utilisées dans un modèle simple permettant de construire les tables habituelles. Ces tables seront améliorées dans les prochaines années à partir de nouvelles mesures effectuées dans le réseau de placettes temporaires.
\end{abstract}

\section{INTRODUCTION}

A l'occasion de la publication d'une table de production provisoire pour l'Épicéa commun (Picea abies (P.)) dans le nord-est de la France, il nous a semblé utile de résumer et de discuter la méthode utilisée pour la construction de ces tables.

Rappelons que la station de Sylviculture et de Production du C.N.R.F. a commencé et poursuit actuellement, la construction de tables de production provisoires pour les principales essences forestières utilisées en France. Ces tables provisoires, assez sommaires, sont construites à partir de premières séries de mesures effectuées dans des placettes temporaires, sans mesure directe d'accroissement et notamment sans sondage systématique à la tarière de Pressler. C'est ce qui contribue à en faire l'originalité.

Ce premier jeu de tables - les premières publiées en France - a surtout pour but 
de combler un vide et de mettre rapidement à la disposition des utilisateurs un outil de gestion qui leur faisait défaut. Avec les secondes, puis les troisièmes séries de mesures effectuées dans les placettes, travail déjà très avancé, une seconde génération de tables de production, plus précises et plus perfectionnées pourra voir le jour.

Nous commencerons par décrire la méthode de façon succincte afin d'en mieux dégager le principe. Pour plus de détail, on pourra se reporter aux explications accompagnant les publications des premières tables.

On étudiera ensuite l'intérêt de la méthode, mais aussi ses limites ainsi que certains problèmes posés par sa mise en œuvre et par la vérification de sa validité.

\section{1. - EXPOSÉ DE LA MÉTHODE}

Les tables de production du C.N.R.F. sont construites à partir d'un nombre de placettes temporaires suffisant pour couvrir tout l'éventail des classes d'âge et des conditions de station. Ces placettes sont utilisées dès la première série de mesures.

On établit, à partir des placettes, un certain nombre de relations mathématiques décrivant les peuplements.

La Sylviculture pratiquée est définie également par un certain nombre de relations.

Les deux séries de relations sont utilisées dans un modèle mathématique, permettant de construire les tables.

Reprenons ces différents points.

\section{1. - Description des peuplements}

On utilise les variables descriptives habituelles *, tirées des mesures effectuées dans les placettes temporaires :

Age

Hauteur dominante

Hauteur moyenne

Circonférence moyenne

Circonférence dominante

Surface terrière

Volume sur pied

Nombres de tiges sur pied
A Volume de l'arbre moyen du peuple-

$\mathrm{H} \varnothing \quad$ ment principal VG

HG Volume de l'arbre moyen enlevé en

$\mathrm{CG}$ éclaircie $\mathrm{v}_{\mathrm{m}}, \mathrm{n}, \mathrm{g}$

$\mathrm{C} \varnothing \quad$ Indice de productivité de la station I

G Nombre de tiges enlevées en éclaircie

$\mathrm{V}$ et surface terrière de ces tiges $\mathrm{n}, \mathrm{g}$

L'ensemble des relations qui lient entre elles ces variables ${ }^{1}$ définit « la structure » des peuplements forestiers de l'essence étudiée dans une région bien définie. Ces relations sont, ou bien des liaisons statistiques calculées par la méthode des régressions multiples progressives, ou bien des conséquences immédiates des définitions (Ex. : $\mathrm{G}=\mathrm{N} \times \mathrm{CG}^{2} / 4 \pi$ ). 1969).

* Pour des définitions plus précises, voir les Annales des Sciences Forestières (DEcourt 1965, 1967,

1. A l'exclusion de $v_{m}, n, g$. 
Dans ce qui suit et sur le schéma ci-joint, toute liaison sera représentée seulement par deux parenthèses. Ainsi, $\mathrm{CG}(\mathrm{HG}, \mathrm{N})$ signifiera qu'on peut déterminer $\mathrm{CG}$ de façon satisfaisante, à partir de $\mathrm{HG}$ et de $\mathrm{N}$, à l'aide d'une équation ou d'un tableau à deux entrées.

Si le matériel de base est fourni par des peuplements éclaircis de façons très variées (en intensité, à plus ou moins longue rotation, par le haut ou par le bas, etc.) et si les placettes sont installées indifféremment juste avant, juste après ou n'importe quand entre les éclaircies, il en résulte que les relations établies sont valables indépendamment du traitement subi par les peuplements.

La pratique a montré qu'il était possible de définir deux ensembles de relations indépendants.

- Le premier est constitué par des relations moyennes indépendantes, à la fois, de l'âge, de l'indice de productivité et des éclaircies pratiquées. Il comprend les relations suivantes :

$$
[\mathrm{S}]\left\{\begin{array}{l}
\mathrm{CG}(\mathrm{HG}, \mathrm{N}) \\
\mathrm{G}(\mathrm{CG}, \mathrm{N}) \\
\mathrm{V}(\mathrm{HG}, \mathrm{G}, \mathrm{N}) \\
\mathrm{HG}(\mathrm{H} \varnothing, \mathrm{N}) \\
\mathrm{C} \varnothing(\mathrm{CG}, \mathrm{N}, \mathrm{HG})
\end{array}\right.
$$

Cet ensemble [S] décrit une structure (au sens précisé plus haut) caractérisant les peuplements étudiés quel que soit leur âge, la station ou la sylviculture qu'ils subissent. Cela signifie que, dans toutes ces conditions et quelles que soient les valeurs que prennent les variables CG, HG, N, etc., les relations (1), (2), (3), (4), (5), restent valables.

On constate, d'autre part, que l'état de l'ensemble [S] est bien défini si on connaît HG et $\mathrm{N}$, ou $\mathrm{H} \varnothing$ et $\mathrm{N}$.

[S] n'est invariant, bien sûr, que dans la limite des observations effectivement faites sur le terrain. En particulier, [S] n'est vérifié que dans l'éventail des sylvicultures effectivement pratiquées dans les placettes de mesures.

- Le second ensemble de relations ne dépend au contraire que des conditions de milieu et de l'âge, indépendamment des éclaircies pratiquées. Il se ramène souvent à une seule relation :

$$
\text { [M] } \mathrm{H} \varnothing(\mathrm{A}, \mathrm{I}) *(1)
$$

La structure [M] ainsi définie caractérise, en somme, la part de l'évolution des peuplements qui échappe à l'action du sylviculteur. Remarquons, au passage, que [M] peut être modifié par l'homme, par l'intermédiaire de pratiques culturales telles que labours ou fertilisations. Dans ce cas, il serait intéressant de savoir si [S] reste invariant ou non. [M] contient d'ailleurs également la variabilité génétique. Il serait également important de savoir si, lorsqu'on change de matériel végétal, [S] reste ou non invariant, seul [M] étant modifié **. Des études comme celles de A. NANSON (1967), sur l'expérience internationale de comparaison de provenance des graines d'Épicéa commun, montrent que cette hypothèse est

\footnotetext{
* Notamment lorsque I est le «Site Index » des auteurs américains, c'est-à-dire la valeur prise par $\mathrm{H} \varnothing$ pour un âge de référence donné (50 ans par exemple).

** C'est ce qui se passe si un changement de provenance modifie la croissance en hauteur sur une même station, sans modifier le tarif utilisable pour une hauteur donnée, ni la production totale correspondant à une même hauteur.
} 
plausible. Sa vérification systématique permettrait de grosses économies dans la mesure des essais de provenance ou de fertilisation, comme également dans l'adaptation des tables de production à des peuplements fertilisés ou réalisés à partir de graines sélectionnées.

Notons, d'une part, que [S] ne contient pas A et I, et, d'autre part, que l'indépendance de $H \varnothing$ vis-à-vis des éclaircies est un fait reconnu par toutes les Stations de Recherches forestières. D’autres variables bénéficient peut-être de propriétés semblables.

Dans ce cas, [M] pourrait comporter plus d'une relation. Si X est une telle variable, on pourrait avoir par exemple :

$$
[\mathrm{M}]\left\{\begin{array}{l}
\mathrm{H} \varnothing(\mathrm{A}, \mathrm{I}, \mathrm{X}) \\
\mathrm{X}(\mathrm{A}, \mathrm{I})
\end{array}\right.
$$

Il est clair, également, que l'état de [M] est bien défini lorsqu'on se donne A et $\mathrm{I}$.

\section{2. - Description de la sylviculture pratiquée}

On définira la sylviculture en décrivant soigneusement l'ensemble des éclaircies pratiquées.

On se donnera :

\section{a) Les moments d'intervention.}

Ces « dates » d'intervention ne sont pas forcément données en fonction de l'âge. On peut parfaitement décider d'intervenir lorsque la hauteur (moyenne ou dominante) a cru d'une quantité donnée. On éclaircira par exemple toutes les fois que la hauteur dominante s'est accrue de $3 \mathrm{~m}$ depuis la dernière éclaircie *. Les rotations [R] sont donc, soit fonctions de $\mathrm{H} \varnothing$ (ou de $\mathrm{HG}$ ), $\mathrm{R}(\mathrm{H} \varnothing)$ ou $\mathrm{R}(\mathrm{HG})$, soit fonctions de l'âge $\mathrm{A}, \mathrm{R}(\mathrm{A})$, selon le désir du sylviculteur.

b) L'intensité des interventions.

Il s'agit du nombre des tiges n enlevées par chaque éclaircie. En fait, nous préférons, ce qui revient au même, nous donner le nombre de tiges $\mathrm{N}$ à laisser sur pied lorsque le peuplement atteint une hauteur dominante donnée ("Stammzahlhaltung » des auteurs allemands). Cette façon de définir l'intensité de l'intervention par une relation continue $\mathrm{N}(\mathrm{H} \varnothing)$, indépendante de $\mathrm{R}(\mathrm{H} \varnothing)$, permet de dissocier l'intensité de la sylviculture pratiquée caractérisée par $\mathbf{N}(H \varnothing)$ et sa brutalité caractérisée par $\mathrm{R}(\mathrm{H} \varnothing)$. Un exemple typique de fixation de l'intensité de l'éclaircie par ce moyen est donné par l'utilisation du facteur d'espacement de Hart-Becking (1953).

c) La nature des éclaircies.

On la caractérisera en comparant les tiges enlevées aux tiges laissées sur pied. Plus précisément, nous la définissons par un facteur $\mathrm{K}$ rapport du volume de l'arbre moyen enlevé en éclaircie, au volume de l'arbre moyen du peuplement sur pied avant éclaircie.

$$
\mathrm{K}=\frac{\mathrm{Vm}}{\mathrm{VG}}
$$

* On peut travailler aussi en hauteur relative. 
Ce rapport est analogue à celui proposé par Hiley W. E. et repris par Delvaux J. (1961) qui rapporte le volume de l'arbre moyen enlevé en éclaircie au volume de l'arbre moyen du peuplement après éclaircie.

On peut se fixer K, en fonction de $\mathrm{H} \varnothing$ (ou de HG) ou en fonction de N. Pour une intensité d'éclaircie donnée, au sens défini ci-dessus, l'éclaircie est d'autant plus «par le haut» que $\mathrm{K}$ est plus grand. En fait, la nature de l'éclaircie ne saurait être rigoureusement indépendante de l'intensité de celle-ci. A partir d'une certaine intensité, on travaille forcément dans l'étage dcminant, même si on se proposait de faire une éclaircie par le bas. Inversement, si on se proposait de faire une éclaircie par le haut, à partir d'une certaine intensité, on enlèvera forcément des tiges dominées, si on ne veut pas déraisonnablement sacrifier des sujets d'avenir. Il arrivera donc, pour certains types de sylviculture, qu'on pourra fixer $\mathrm{K}$ à partir de $\mathrm{H} \varnothing$ et de $\mathbf{N}$ :

$$
\mathrm{K}(\mathrm{H} \varnothing, \mathrm{N})
$$

En résumé, l'intervention du sylviculteur est bien définie par un ensemble [E] de relations décrivant sans ambiguïté les éclaircies pratiquées.

$$
\begin{aligned}
& {[\mathrm{E}]\left\{\begin{array}{l}
\mathrm{N}(\mathrm{H} \varnothing) \\
\mathrm{R}(\mathrm{H} \varnothing) \\
\mathrm{K}(\mathrm{H} \varnothing, \mathrm{N})
\end{array}\right.} \\
& {\left[\mathrm{E}^{\prime}\right]\left\{\begin{array}{l}
\mathrm{N}(\mathrm{H} \varnothing) \\
\mathrm{R}(\mathrm{A}) \\
\mathrm{K}(\mathrm{H} \varnothing, \mathrm{N})
\end{array}\right.}
\end{aligned}
$$

[E] est indépendant de l'âge, [E'] dépend au contraire de l'âge. Le choix de $[E]$ ou de $\left[E^{\prime}\right]$ peut se faire plus ou moins intuitivement à partir de l'observation des placettes de mesures, ou bien aussi à l'aide des tables de production déjà existantes, ou mieux à partir de dispositifs de comparaison des éclaircies et en fonction d'un objectif économique donné, caractérisé, par exemple, par l'état dendrométrique final souhaité.

On peut également envisager de définir [E] en optimisant une fonction économique, par les méthodes de la Recherche opérationnelle, notamment la programmation dynamique.

\section{3. - Modèle pour construire la table de production}

On suppose que les relations définissant [S] et [M] peuvent être établies avec une précision satisfaisante *. Dans les tables déjà parues, et également pour l'Épicéa dans le nordest de la France, on constate effectivement que l'âge A n'intervient pas de façon significative dans les équations de régression définissant [S]. Il s'agit là, d'ailleurs, d'une vérification partielle de la loi de Eichborn, (en l'absence d'éclaircie et pour une densité de plantation donnée, le volume produit ne dépend que de la hauteur). L'ensemble [E], lui, est donné par le sylviculteur.

Le calcul se déroule alors selon le schéma ci-joint. [S] donne une description de base des peuplements indépendamment du temps (de l'âge), de la station, de la sylviculture. La

* Voir l'exemple donné en annexe. Voir également l'article suivant dans ce même numéro des Annales : « Tables de production provisoires pour l'Épicéa Commun dans le Nord-Est de la France ». 
connaissance de [E] permet de définir [S] à partir de $\mathrm{H} \varnothing$ et de $\mathrm{N}$. On obtient ainsi une table intermédiaire * décrivant les états successifs pour des hauteurs croissantes des peuplements soumis à la sylviculture [E] et cela quel que soit leur âge et la classe de productivité de la station. Les variations de HG avec le type et l'intensité de l'éclaircie sont calculées, en imposant la condition d'invariance de [S] au peuplement avant l'éclaircie et après l'éclaircie. En effet, l'enlèvement de $n$ tiges et d'un volume $v$, impose pour maintenir l'invariance de [S], une certaine variation de $\mathrm{HG}, \mathrm{CG}$, etc. De plus, $[\mathrm{S}]$ reste vérifiée pour tous les états successifs correspondant aux lignes de la table.

SCHÉMA DE CONSTRUCTION DES TABLES DE PRODUCTION

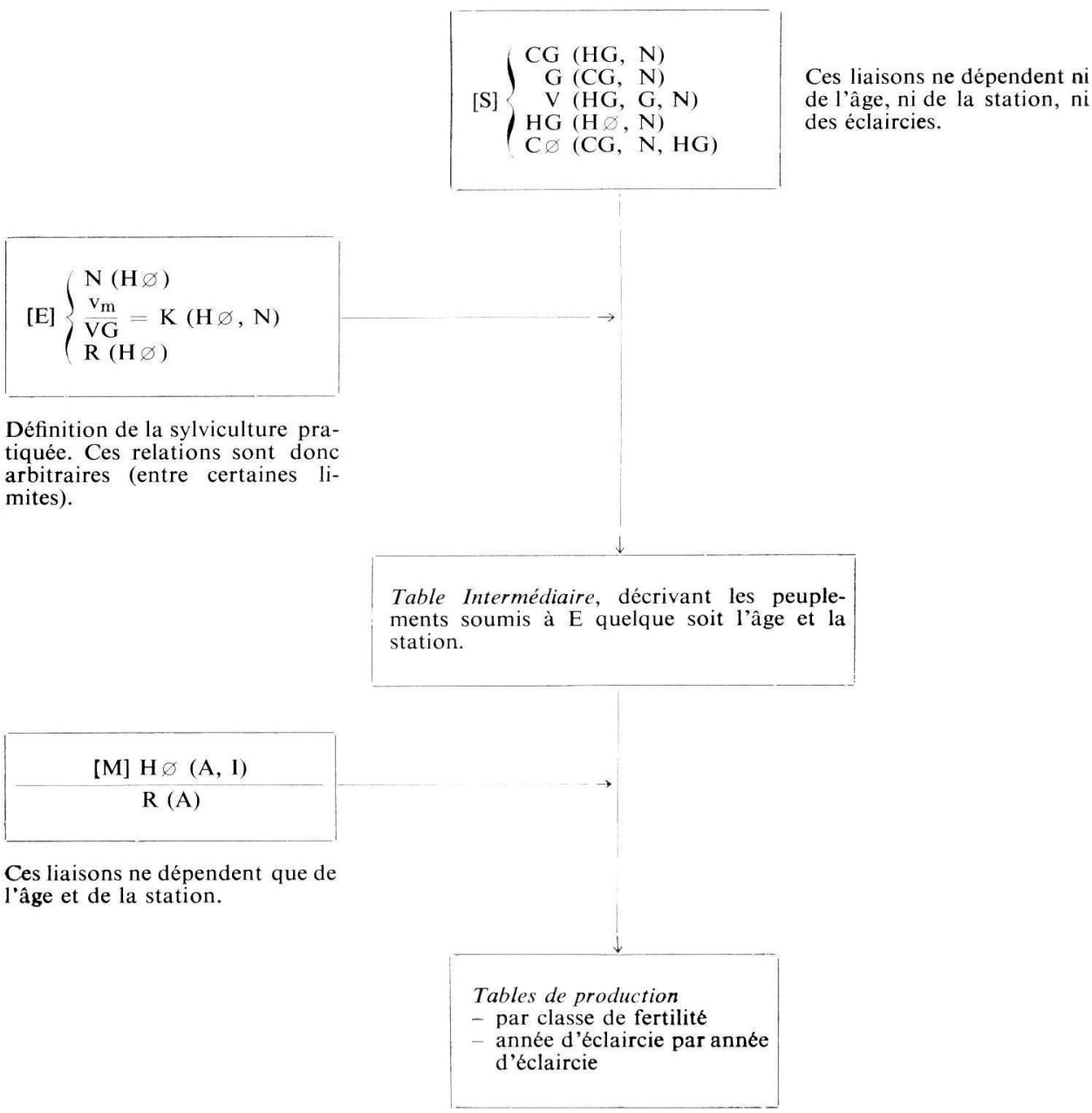

* Analogue à la table de base des Auteurs anglais. 
Les tables de production de type classique, c'est-à-dire, établies par classes de productivité et pour des âges croissants des peuplements, sont obtenues à partir de cette table intermédiaire et des relations

$$
\left\{\begin{array}{l}
\mathrm{H} \varnothing(\mathrm{A}, \mathrm{I}) \\
\mathrm{R}(\mathrm{A})
\end{array}\right.
$$

par simple interpolation linéaire dans la table intermédiaire.

Ce processus implique que la définition des rotations à partir de l'âge (à partir de $\mathrm{R}$ (A)) ne modifie pas les résultats de la table intermédiaire où les relations sont définies à partir de la hauteur (à partir de $\mathrm{R}(\mathrm{H} \varnothing)$ ). L'allure de la croissance ne doit pas être modifiée, toute chose égale par ailleurs, par un changement dans la durée des rotations. Cette hypothèse nous semble admissible si on choisit pour construire la table intermédiaire des hauteurs successives assez proches. (Nos tables font en général varier la hauteur de $3 \mathrm{~m}$ en $3 \mathrm{~m}$ ce qui donne des rotations moyennes en âge de l'ordre de 5 à 10 ans).

Cette méthode mise au point en 1964, lors de la construction des tables de production pour le Pin Sylvestre et le Pin Laricio en Sologne, a été réutilisée pour le Douglas, le Pin Maritime et l'Épicéa commun. Le processus d'établissement des tables fait l'objet d'un programme (FORTRAN IV), les relations de [S] et [M] font l'objet de « subroutines » tandis que celles de [E] sont fournies dans les données de calcul selon la sylviculture désirée.

\section{II. - INTÉRÊT DE LA MÉTHODE}

\section{1. - Généralité de la méthode}

Afin de construire rapidement une première " génération 》 de tables de production, même imparfaites, nous avons simplifié au maximum la description des peuplements, c'està-dire, l'ensemble [S]. La relation $\mathrm{HG}(\mathrm{H} \varnothing)$ notamment n'est pas rigoureusement indépendante des éclaircies pratiquées, puisque $\mathrm{HG}$ varie avec l'éclaircie et que $\mathrm{H} \varnothing$ est parfaitement indépendant. Cependant la liaison HG $(H \varnothing)$ est tellement étroite qu'en première approximation nous l'avons retenue comme telle (voir tableau ci-après). Cela entraîne la possibilité de travailler indifféremment avec $\mathrm{H} \varnothing$ ou avec $\mathrm{HG}$, pour la construction d'un premier jeu de tables provisoires.

\begin{tabular}{l|c}
\hline \multicolumn{1}{c|}{ HG $(\mathrm{H} \varnothing)$} & $\mathrm{R}^{2}$ \\
\hline & \\
Pin Sylvestre & 0,98 \\
Pin Laricio & 0,97 \\
Pin Maritime & 0,99 \\
Douglas & 0,98 \\
Épicéa & 0,97 \\
\hline
\end{tabular}

La méthode peut donc être affinée, en introduisant de nouvelles relations dans [S]. On pourrait, par exemple, tirer de nos placettes, qui contiennent chacune de 20 à 30 arbres échantillons, une relation donnant la hauteur de chaque tige (HT) en fonction de sa circonférence $\mathrm{C}$ et d'autres variables du peuplement :

$$
\text { HT (C, HG, N...) }
$$


Celà permettrait, connaissant la répartition en circonférence des tiges, qui serait à définir dans [E], de calculer le volume $\mathrm{V}$ à partir d'un tarif à deux entrées classiques $\mathrm{HT}$ et $\mathrm{C}$ et également d'apprécier exactement la variation instantanée «technique » de HG au moment du passage de l'éclaircie, en fonction des caractéristiques de celle-ci.

\section{2. - Définition objective de la zone de validité des tables}

Nous avions déjà souligné la difficulté pour définir une région géographique «homogène » propre à la construction d'une seule table de production pour une même essence (DECOURT N. 1964). Nous suggérions, alors, d'utiliser la relation liant la production totale à la hauteur dominante, et celle liant la hauteur dominante à l'âge et à l'indice de fertilité. Le problème était de comparer entre elles ces relations établies dans des régions différentes et, en l'absence de différence significative, de regrouper ces régions en une seule.

La méthode exposée ci-dessus donne un moyen de comparaison plus rigoureux. $\mathrm{Si}$ l'ensemble des relations de $[\mathrm{S}]$ et $[\mathrm{M}]$ ne diffèrent pas significativement pour deux régions données, on peut regrouper ces régions en une seule. On est donc amené à comparer une par une les équations de régression figurant dans [S] et [M].

Cette méthode appliquée aux peuplements de Pins maritimes du sud-ouest nous a conduit à construire deux tables de production : une pour les Landes, une autre pour les dunes. Inversement, dans le nord-est de la France et pour l'Épicéa commun, une seule table pourra convenir pour les basses-Vosges et pour les plateaux calcaires.

\section{3. - Extension de la méthode}

Le mode de comparaison, évoqué ci-dessus et effectivement mis en œuvre pour la délimitation de zones de croissance suffisamment homogènes, peut également s'appliquer aux comparaisons d'essences.

Deux essences ayant des ensembles de relations $[\mathrm{S}]$ et $[\mathrm{M}]$ non significativement différents, pourraient ainsi avoir des tables de production communes. Ces tables seraient seulement en parties communes, si [E] devait être nécessairement différent pour l'une et pour l'autre en vertu de considérations technologiques.

On peut évoquer ici la possibilité de construire un seul jeu de tables applicables à la plupart des espèces et dans lequel on pourrait choisir la table convenable pour un peuplement donné, d'une espèce donnée, sur une station donnée, à partir de variables caractérisant le milieu ou le peuplement.

Cela conduit évidemment à rechercher un ensemble de relations valables pour tous les peuplements indépendamment de l'essence, de la localisation géographique, de la productivité de la station, de l'âge et de la sylviculture pratiquée. Ce premier ensemble décrirait un certain nombre de lois générales de la production des peuplements forestiers concernés. Un second ensemble de relations ferait intervenir, au contraire, des conditions de milieu, l'essence et l'âge du peuplement, la sylviculture pouvant être décrite comme ci-dessus par un troisième jeu de relations. Ce problème sort du cadre de cette note, mais le projet ne semble pas utopique à priori. 


\section{4. - Utilisation pour des aménagements}

Un des aspects intéressants de cette méthode, utilisant des mesures tirées de placettes temporaires, est de ne pas faire appel à des estimations de l'accroissement courant, à l'aide par exemple de sondages à la tarière de Pressler, comme la plupart des méthodes à base de placettes temporaires.

Ces estimations, pour être précises, nécessitent un grand nombre de sondages et sont donc très coûteuses. De plus, elles laissent des traces sous formes de trous dans les billes de pied. Cela explique, du reste, qu'à l'origine, nos placettes étant installées la plupart du temps en forêt privée, nous ayons dû nous passer de ces sondages et modifier en conséquence la méthodologie habituelle de construction des tables à partir de placettes mesurées une seule fois.

La très grande simplicité des mesures nécessaires permet d'envisager l'utilisation de cette méthode pour construire des tables de production valables pour un massif forestier déterminé, ou pour une région, à partir d'un inventaire statistique d'Aménagement de type classique, moyennant quelques mesures supplémentaires.

Ces mesures seraient peu coûteuses, en tous cas moins que des sondages systématiques à la tarière.

\section{III. - LIMITES D'UTILISATION DU MODÈLE}

\section{1. - Problème du choix d'une sylviculture « à priori ».}

Il va de soi qu'on ne peut utiliser les relations de [M] et de [S] en dehors des limites des observations qui ont permis de les établir. On ne pourra donc pas choisir une sylviculture sortant du cadre des sylvicultures effectivement pratiquées dans les placettes servant de base à la construction de la table.

Dans une même région et pour une même essence, on peut cependant trouver un jeu de placettes correspondant à des interventions très différentes, en intensité notamment, allant de peuplement non éclaircis jusqu'à des peuplements très fortement éclaircis. Le problème qui se pose est de savoir si, entre ces limites, il est licite de choisir, à priori, n'importe quelle sylviculture pour construire la table.

Dans notre modèle, la sylviculture est bien définie, si on se donne :

- les âges d'intervention $\mathrm{R}$ (A)

- la relation $\mathrm{N}(\mathrm{H} \varnothing)$, ou $\mathrm{N}(\mathrm{HG})$

- les valeurs de $\mathrm{K}$ à chaque intervention, par exemple sous la forme d'une relation $\mathrm{K}(\mathrm{H} \varnothing)$ ou $\mathrm{K}(\mathrm{HG})$.

Le passage de la «table intermédiaire » (indépendante de A) aux tables classiques, présentées par classe de fertilité et où chaque ligne correspond à un âge d'intervention, a déjà été discuté plus haut $(\$ 1,3)$. 
La variable $\mathrm{N}$ intervient explicitement dans les équations de [S]. L'évaluation de $\mathrm{N}$ est du reste facile à faire dans les placettes. Il est donc licite dans les limites rappelées plus haut, de fixer arbitrairement N. Il semble donc qu'on puisse faire varier assez largement l'intensité des éclaircies, et, ainsi, construire des tables de production pour différente intensité d'éclaircie fixée à l'avance.

Le coefficient $\mathrm{K}$, qui - rappelons-le - définit la nature de l'éclaircie, n'intervient pas explicitement dans les équations de [S] et cela, faute de mesure directe de ce coefficient dans les placettes. Les équations de [S] sont donc valables uniquement pour un coefficient $\mathrm{K}$ moyen qui caractérise le type moyen d'éclaircie pratiquée dans l'ensemble des placettes de mesures. Comme pour les rotations $\mathrm{R}$ (A), le choix arbitraire de $\mathrm{K}$ suppose que si on l'avait effectivement mesuré, ce coefficient n'interviendrait pas dans les équations de [S]. Notre modèle implique cette hypothèse un peu risquée. Mais de plus, comme le champ de variation de $\mathrm{K}$ dans l'ensemble des placettes n'est pas connu, il importe d'être prudent et de ne pas trop s'éloigner des $\mathrm{K}$ moyens. On peut estimer ceux-ci, soit par des observations directes, notamment dans les placettes permanentes, ou dans les placettes temporaires ou l'éclaircie vient de passer, soit à partir de tables de production étrangères correspondant à des sylvicultures voisines de celle pratiquée dans la région étudiée. Il serait hasardeux d'utiliser pour l'Épicéa commun du nord-est de la France, par exemple, traditionnellement éclairci par le bas, des coefficients K correspondants aux « Management Tables» britanniques qui caractérisent des interventions par le haut très marquées.

Ces considérations expliquent le choix unique et prudent de $\mathrm{K}$ dans les tables déjà publiées.

\section{2. - Évaluation de la production totale}

L'évaluation de la production totale est rendue possible par le modèle proposé. Il faut, cependant, en toute rigueur, signaler une cause d'erreur possible. Les équations de [S] sont calculées, - c'est le principe même de la méthode —, à partir de placettes établies dans des peuplements « avant-éclaircie » ou « après éclaircie » indifféremment. Pour une même sylviculture, il n'est pas exclu que le caractère " avant-éclaircie » ou " après-éclaircie », si on en avait tenu compte, aurait modifié les équations de $\mathrm{S}$ et notamment la relation $\mathrm{V}$ (HG, $\mathrm{N})$ qu'on peut tirer de [S]. Supposons que cette relation moyenne donne une estimation par défaut du volume sur pied $\mathrm{V}_{\mathrm{Av}}$, avant-éclaircie, et par excès du volume $\mathrm{V}_{\mathrm{AP}}$, aprèséclaircie et supposons même, pour simplifier, que l'écart en plus " après » soit égal à l'écart en moins « avant ».

Soit $d$ cet écart, on aura :

$$
\text { Estimation de } \begin{aligned}
\mathrm{V}_{\mathrm{AV}} & =\mathrm{V}_{\mathrm{AV}}-d, \\
-\quad \mathrm{V}_{\mathrm{AP}} & =\mathrm{V}_{\mathrm{AP}}+d .
\end{aligned}
$$

L'éclaircie $v$ sera estimée $(v-2 d)$ et la production totale $\left(V_{A P}+v\right)$ sera estimée $\left(V_{A P}+\right.$ $\mathrm{v}-\mathrm{d})$. Ainsi l'application d'une relation moyenne V $(\mathrm{HG}, \mathrm{N})$, entraînera, à hauteur et nombre de tiges égaux, une sous-estimation de la production totale.

Notons cependant que cette erreur dépend de la précision de l'ajustement de V ( $\mathrm{HG}, \mathrm{N})$. Si cet ajustement est très bon, avec, par exemple, des écarts moyens de l'ordre de $\pm 10 \%$ 
de la valeur ajustée, l'erreur éventuelle, signalée ci-dessus, a peu de chance de dépasser cette valeur, d'autant plus que d'autres causes de variations doivent « tenir » dans cette frange d'incertitude.

Il faut cependant noter que les erreurs à craindre sur l'évaluation des éclaircies lesquelles sont calculées par différence — sont évidemment beaucoup plus fortes.

\section{IV. - PROBLÈME DE L'AJUSTEMENT DES ÉQUATIONS}

\section{1. - Ajustement des relations de [S]}

Notons que les relations de [S] et de [M] sont utilisées en fait successivement, alors que celles de [E] sont arbitraires (dans certaines limites). Les relations de [S] sont des liaisons linéaires entre des fonctions des paramètres descriptifs dendrométriques habituels :

par exemple : $\mathrm{V}=a+b f(\mathrm{G})+c . g(\mathrm{HG})$

Elles sont ajustées séparément par la méthode des moindres carrés. Ce procédé est correct et ne nécessite pas la résolution d'un système d'équations simultanées, dès lors qu'on remarque que le modèle proposé est un modèle récursif*. En effet, les liaisons entre les variables endogènes apparaissant dans [S] sont de type causal, les équations s'enchaînent les unes aux autres de façon logique. On peut décrire la structure [S] par un réseau de flèches qui aurait l'allure suivante :

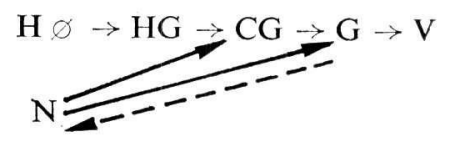

Ce modèle (en trait plein) s'oppose à celui qu'on obtiendrait s'il était nécessaire de prévoir les liaisons à double sens telle que celle symbolisée par la flèche en pointillé du schéma ci-dessus.

Dans le cas des modèles récursifs, on démontre que la meilleure estimation des équations du modèle peut être obtenue par l'ajustement séparé de chacune de ses équations.

\section{2. - L'ajustement des relations de [M]}

[M] se réduit en fait dans nos tables actuelles à une seule relation $\mathrm{H} \varnothing(\mathrm{A}, \mathrm{I})$ ou éventuellement et de façon plus approximative HG (A, I). Il existe plusieurs méthodes pour obtenir ces relations. Ces méthodes se ramènent à trois types principaux :

- ajustement direct à partir du nuage de points obtenus en portant $\mathrm{H} \varnothing$ (ou HG) en ordonnée et $\mathrm{A}$ en abcisse. On prend alors comme « courbe guide » la courbe moyenne et on

* Voir un exposé plus complet de ce problème dans « liaisons stochastiques en économie » Jean Dubos, Dunod, 1971. 
déduit les autres courbes du faisceau défini par la relation $H \varnothing(A, I)$, en tenant compte de la dispersion des points autour de la courbe guide (BRUCE O. et Schumacher F., 1950; CantiaNi M. et Bernetti G., 1962; Decourt N., 1965). On peut aussi prendre comme « courbes guides » les courbes limitant le nuage de points vers le haut et vers le bas (DECOURT N., 1967);

- utilisation d'analyse de tiges prélevées dans l'étage dominant. Cette méthode, déjà ancienne, est fréquemment utilisée pour la construction d'un faisceau de « Site Index curves » qui correspond à notre relation $\mathrm{H} \varnothing(\mathrm{A}, \mathrm{I})$ (voir par exemple CurTis R.-O., 1964). Elle suppose, ce qui paraît du reste vérifié, que les tiges dominantes prélevées au moment de la mesure soient restées dominantes pendant toute la vie du peuplement et que leur croissance n'ait pas été modifiée par la sylviculture pratiquée;

- utilisation d'un certain nombre d'évaluations de l'accroissement courant en hauteur, obtenues, par exemple, par la mesure des derniers verticilles d'arbres-échantillon. On essaie alors d'ajuster une équation différentielle rendant compte des liaisons entre la hauteur, l'âge et l'accroissement courant en hauteur :

$$
f\left(\mathrm{H} \varnothing^{\prime} \mathrm{A}, \mathrm{A}, \mathrm{H} \varnothing\right)=\mathrm{O}
$$

Par intégration, on obtient une équation $\mathrm{H} \varnothing=g(\mathrm{~A}, \mathrm{C})$, la constante $\mathrm{C}$ pouvant s'exprimer en fonction de l'indice de productivité I tel que nous l'avons défini (valeur de $\mathrm{H} \varnothing$, pour un âge de référence donné).

Ces trois méthodes peuvent être utilisées simultanément. Dans le cas de Pin maritime dans le sud-ouest de la France, les résultats obtenus ainsi par des moyens très différents ont été tout-à-fait concordants (Decourt N. et Lemoine B., 1969).

\section{V. - VÉRIFICATION DU MODÈLE}

En l'absence de données nombreuses issues de placettes permanentes et permettant de comparer les résultats trouvés à des mesures de production effectivement faites sur le terrain, les vérifications sont difficiles.

Le seul essai que nous avons pu tenter porte sur deux stations de Pin maritime de Dunes (Hourtin et Ste Eulalie). Nous avons utilisé les mesures effectuées dans 7 placettes, soit au total de 30 à 40 estimations de différents paramètres dendrométriques, y compris la production totale, la production moyenne annuelle et l'accroissement courant annuel en volume. Utilisant les équations calculées pour la table de production, on a, pour chaque placette, construit une table de production spéciale correspondant à la sylviculture effectivement appliquée dans la placette.

La comparaison des écarts relatifs entre valeurs observées dans les peuplements et valeurs calculées a donné les résultats suivants : 


\begin{tabular}{|c|c|c|c|}
\hline $\begin{array}{c}\text { Paramètres } \\
\text { étudiés }\end{array}$ & $\begin{array}{l}\text { Nombre de } \\
\text { comparaisons }\end{array}$ & $\begin{array}{c}\text { Biais : } \\
\text { Moyenne des écarts } \\
\text { (valeur calculée moins } \\
\text { valeur observée) } \\
(\text { en } \%)\end{array}$ & $\begin{array}{c}\text { Écart moyen } \\
\text { autour } \\
\text { du biais } \\
\text { (en } \% \text { ) }\end{array}$ \\
\hline $\mathrm{HG}$ & 23 & $\begin{array}{l}+\quad 0,83 \\
\end{array}$ & 2,29 \\
\hline CG & 39 & $-\quad 2,25$ & 6,72 \\
\hline G & 39 & $+\quad 0,81$ & 11,51 \\
\hline V & 39 & $-\quad 0,23$ & 14,78 \\
\hline $\begin{array}{c}\mathrm{V} \\
\text { Production }\end{array}$ & 29 & $-\quad 7,48$ & 42,69 \\
\hline totale & 39 & $-\quad 4,93$ & 14,16 \\
\hline $\begin{array}{l}\text { Accroissement } \\
\text { courant annuel }\end{array}$ & 35 & $+\quad 10,82$ & 25,00 \\
\hline $\begin{array}{l}\text { Accroissement } \\
\text { moyen annuel }\end{array}$ & 39 & $-4,25$ & 15,01 \\
\hline
\end{tabular}

Ces comparaisons concernant 7 placettes, traitées de façon très différentes, mais seulement deux stations, montrent bien que, si les erreurs sur une estimation sont fortes, l'estimation par le modèle est en moyenne correcte avec, cependant, une sous-estimation de la production moyenne d'environ $5 \%$ et une sur-estimation de la production courante de l'ordre de $10 \%$.

Reçu pour publication en octobre 1971

\section{SUMMARY}

\section{METHOD USED TO CONSTRUCT A FIRST SET OF PROVISIONAL YIELD TABLES IN FRANCE}

The method used temporary plots data without measurement of volume increment. The stands, the site index curves and the sylvicultural treatment are described by mathematical relations. These relations are used in a simple model alloving to construct usual yield tables. These tables will be improved in the next years on the ground of new measures made in the network of temporary plots.

\section{ZUSAMMENFASSUNG}

\section{DIE IN FRANKREICH ANGEWANDTE METHODE ZUR RASCHEN} ERSTELLUNG VON VORLAUFIGEN ERTRAGSTAFELN.

Die Methode verwendet die Daten einmalig aufgenommener Probeflächen wobei jedoch der Massenzuwachs nicht direckt gemessen wird. Die Bestände und auch das Höhenwachstum in Abhängigkeit vom Standort, werden durch mathematische Beziehungen beschrieben.

Die waldbauliche Behandlung wird auf dieselbe Art und Weise beschrieben. Die mathematischen Beziehungen werden in einem einfachen Modell verwendet, das die Aufstellung der üblichen Ertragstafeln erlaubt. An Hand von wiederholten Aufnahmen der Probeflächen werden die Tafeln in den kommenden Jahren verbessert werden.

\section{RÉFÉRENCES BIBLIOGRAPHIQUES}

BECKING J. H., 1953. Einige Gesichtpunkte für die Durchförstung von vergleichenden Durchforstunversuchen in gleichälteringen Beständes. C.R. 11e congrès de l'I.U.F.R.O., p. 580-582. 
Bruce O et Schumacher F., 1950. Forest Mensuration. Mc Graw, Mill Book Company, New-York, 1950. Cantiani M. et Bernetti G., 1962. Tavola alsometrica delle Abetine Coetanee della Toscana. Firenze 1962. Curtis R. O., 1964. A Stem-Analysis approach to Site. Index curves. Forest. Sci., 10 (2), 241-256.

Decourt N., 1964. Les tables de production, leurs limites et leur utilité. R.F.F., août-septembre 1964, p. 640.

Decourt N., 1965. Le Pin Sylvestre et le Pin Laricio de Corse en Sologne. Tables de production provisoires et méthodes utilisées pour les construire. Ann. Sciences Forestières, 22 (2), 259-318.

Decourt N., 1967. Le Douglas dans le Nord-Est du Massif Central. Tables de production provisoires. Ann. Sciences Forestières, 24 (1), 45-84.

Decourt N. et Lemoine B., 1969. Le Pin maritime dans le Sud-Ouest de la France. Tables de production provisoires. Ann. Sciences Forestières, 26 (1), 3-44.

Delvaux J., 1961. Calcul des éclaircies numériques (adopté et modifié d'après Hiley W. E.) Bulletin de la Société Royale Forestière de Belgique, 68 année, oct. 1961, p. 453-472.

Dubos J., 1971. Liaisons stochastiques en Économie. Dunod, Paris, 1971, 488 pages.

NANSON A., 1964. Données complémentaires au sujet de l'expérience internationale sur l'origine des Graines d'Épicéa en Belgique. Station Recherche Forestière de Groenendaal-Hoeillaart, Travaux. Série $B, \mathrm{n}^{\circ} 28,40$ pages.

\section{ANNEXE}

PIN MARITIME DANS LE SUD-OUEST (LANDES)

(TABLES PUBLIÉES EN 1969)

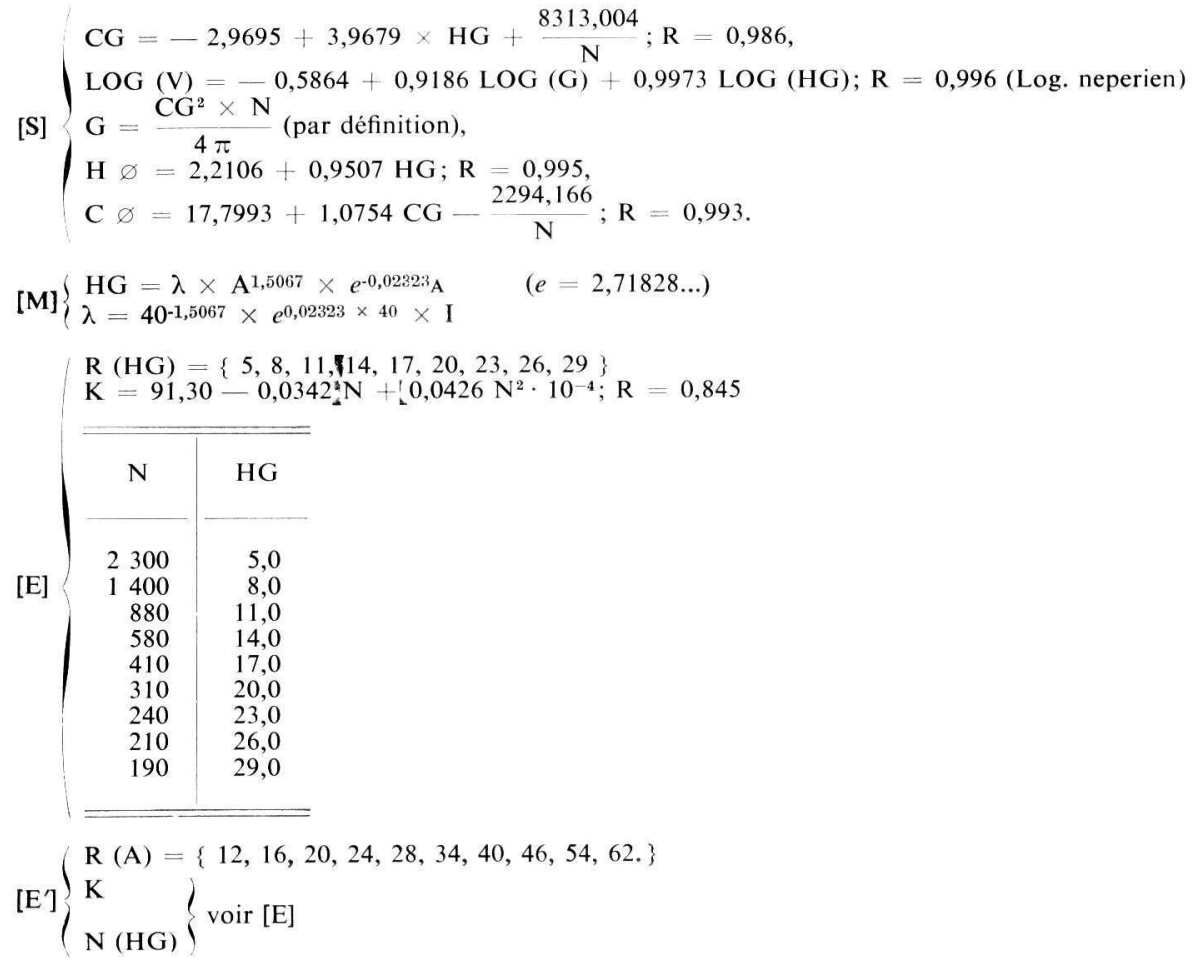

